stimmungen gesteigert werden, für die biologisches Material enteiweißt werden muß. Bei quantitativen Bestimmungen erfordert die Enteiweißung mit denaturierenden Substanzen vor der eigentlichen Reaktion das Abmessen eines Probenaliquots und die Zugabe des Fällungsmittels. Diese beiden quantitativen Schritte, die lange dauern und die Genauigkeit der Bestimmungen mindern, entfallen aber, wenn die Proben diazentriert werden. Die Methode könnte daher klinisch-chemische Routinelaborarbeiten fühlbar entlasten.

Auch ist es möglich, durch Diazentrieren den Anteil eines Stoffes zu bestimmen, der im Serum oder Liquor fest an Eiweiß gebunden ist. Untersuchungen dieser Art, die wir an der Harnsäure des menschlichen Serums begonnen haben, zeigen, daß die Eiweißbindungskapazität des Blutes für solche Stoffe offenbar ein pathophysiologisches Kriterium mancher Krankheitssyndrome ist.

\title{
Literatur
}

1. Campbell, W. R. und M. T. Hanna, J. biol. Chemistry 119, 9 (1937). - 2. Lorentz, K. und H. Hoffmeister, Mikrichimica Acta (Wien) 1062 (1966). - 3. Barthelmar, W. und R. Czok, Klin. Wschr. 40, 585 (1962). - 4. Moore, S. und W. H. Stein, J. biol. Chemistry 211, 907 (1954). - 5. Pfleiderer, G. und T. Wieland, Ann. Acad. Sci. fenn. A II, 60, 381 (1955). - 6. Hamilton, P. B. und D. D. van SLYKe, J. biol. Chemistry 150, 231 (1943). 7. Stern, W. H. und S. Moore, J. biol. Chemistry 211, 915 (1954). -8. Tallan, H. H., S. Moore und W. H. Stern, J. biol. Chemistry 219, 257 (1956). - 9. Beckman Amino Acid Analyzer Model 120 B Instruction Manual, Part 7-1 (1962). - 10. Hamilton, P. B. und R. M. Archibald, Indian Eng. Chem. Anal., Ed. 16, 136
(1944). - 11. Prescott, B. A. und H. Waelsch, J. biol. Chemistry 167, 855 (1947). - 12. Ferry, J. D., Chem. Revs. 18, 373 (1936). 13. Nrcholas, H. O., J. biol. Chemistry 97, 475 (1932). - 14. Rehberg, P. B., Acta Physiol. Scand. 5, 305 (1943). - 15. WaArd, D. J. DE, Arch. néerland. physiol. 2, 530 (1918). - 16. FELdMAN, I., R. A. Danley und J. F. O'Leary, Analytic. Chem. 22, 837 (1950). - 17. Toribara, T. Y., Analytic. Chem. 25, 1286 (1953). - 18. SIEGmund, P., Habilitationsschrift, Freie Universität Berlin, 1962. - 19. Metzner, H., Naturwissenschaften 14, 388 (1953). - 20. Stekelenburg, G. J. und J. Desplanque, Technicon Instruments Company Ltd., $4^{\text {th }}$ Amino Acid Colloq., London, p. 83 (1966).

Dozent Dr. W. Tarnowski

2 Hamburg 20, Martinistraße 52

\section{Vergleichende Untersuchungen über die Antivitamin-B -Wirkung von L- und D-Penicillamin}

\author{
Von F. Körber, G. Hasenbank und P. Siegmund \\ Aus dem Pbysiologisch-Chemischen Institut der Freien Universität Berlin (Direktor: Prof. Dr. Dr. E. Schütte)
}

(Eingegangen am 22. Juni 1967)

Applikation von täglich $10 \mathrm{mg}$ L- bzw. D-Penicillamin intraperitoneal über drei Wochen führt bei Ratten

1. nach Belastung mit $40 \mathrm{mg}$ Tryptophan zu einer Xanthurensäure-Ausscheidung von $5,7 \mathrm{mg}$ nach L-Penicillamin, von $0,6 \mathrm{mg}$ nach D-Penicillamin gegenüber $0,2 \mathrm{mg}$ bei den Kontrolltieren.

2. zu einer 20 proz. Verminderung der Aktivität von Aspartataminotransferase in der Leber nach L-Penicillamin, während nach DPenicillamin kein Unterschied zu den Kontrolltieren auftritt.

3. zu einer 50 proz. Verminderung der Aktivität von Alaninaminotransferase in der Leber nach L-Penicillamin und einer 20 proz. Verminderung nach D-Penicillamin.

Rats received $10 \mathrm{mg}$. of $\mathrm{L}$ - or $\mathrm{D}$-penicillamine daily over a period of three weeks.

1. The excretion of xanthurenic acid after loading with $40 \mathrm{mg}$. tryptophan increased from $0.2 \mathrm{mg}$. in the control animals to $5.7 \mathrm{mg}$. after L-penicillamine and $0.6 \mathrm{mg}$. after D-penicillamine.

2. The aspartate-aminotransferase activity in the liver of L-penicillamine-treated animals was decreased by $20 \%$, while $\mathrm{D}$-penicillamine had no effect.

3. The alanine-aminotransferase activity was decreased by $50 \%$ in the liver after treatment with L-penicillamine, and by $20 \%$ after $\mathrm{D}$ penicillamine.

In einer Reihe von Arbeiten haben DU VIGNEAUd und Mitarbeiter (1-5) gezeigt, daß dem Futter zugesetztes L-Penicillamin das Wachstum von Ratten hemmt. Die Tiere zeigten Hautsymptome, wie sie bei Vitamin- $\mathrm{B}_{6}$ Mangel auftreten und schieden nach Tryptophanbelastung große Mengen Xanthurensäure aus. Die Transaminaseaktivitäten im Leberhomogenat waren vermindert. Durch Vitamin- $\mathrm{B}_{6}$-Gaben konnten diese Wirkungen verhindert werden. Auch für D-Penicillamin, das für die Behandlung der Wrusonschen Erkrankung verwendet wird, ist eine Antivitamin- $\mathrm{B}_{6}$-Wirkung be- schrieben worden (6-8). Eine Untersuchung beider Penicillamin-Antipoden unter vergleichbaren Versuchsbedingungen fehlt bisher jedoch.

\section{Material und Methoden \\ Reagenzien und Referenzsubstanzen}

L- und D-Penicillamin erhielten wir von Heyl und Co. ${ }^{1}$ ), Berlin. Die Reinheit wurde durch Messung der optischen Drehung im lichtelektrischen Polarimeter (Zeiss), durch SH-Gruppentitration mit

1) Wir danken der Firma für die kostenlose Uberlassung dieser Substanzen. 
p-Chlormercuribenzoat nach (9) und chromatographisch nach vorheriger Oxydation mit Perameisensäure zur Sulfonsäure $(10,11)$ kontrolliert. Für die Xanthurensäureeichkurve verwendeten wir Xanthurensäure p. a. der Fa. Fluka, Buchs/Schweiz (Vergleichssubstanz für den Nachweis eines Vitamin- $\mathrm{B}_{6}$-Mangels). L-Aminosäuren, Ketosäuren, Coenzyme und Puffersubstanzen für die Enzymbestimmungen waren analysenreine Präparate der Firmen E. Merck, Darmstadt, C. F. Boehringer Söhne, Mannheim und Serva, Heidelberg. Lactat- und Malatdehydrogenase kauften wir bei Fa. C. F. Bochringer Söhne, Mannheim.

\section{Apparale}

Die photometrischen Messungen wurden mit dem Spektralphotometer PMQ II (Zeiss) und dem Photometer Eppendorf durchgeführt.

\section{Tierversuche}

Weißen männlichen Wistar-Ratten von etwa $250 \mathrm{~g}$ Körpergewicht, die Wasser und käufliches Standardfutter (Altromin R 15) ad libitum erhielten, wurden täglich $10 \mathrm{mg} \mathrm{D}-\mathrm{bzw}$. L-Penicillamin in $2 \mathrm{~m} / 0,9$ proz. $\mathrm{NaCl}-L$ ösung intraperitoneal injiziert. Eine Kontrollgruppe erhielt Futter ebenfalls ad libitum, eine andere jedoch nur $10 \mathrm{~g}$ täglich, um eine zu der Versuchsgruppe, die L-Penicillamin erhielt, vergleichbare Gewichtsabnahme zu erzielen. Die Kontrolltiere erhielten täglich $2 \mathrm{~m} l 0,9$ proz. $\mathrm{NaCl}$-Lösung intraperitoneal. Das Gewicht der Tiere wurde täglich kontrolliert. Nach drei Wochen mit täglichen Penicillamingaben (bei den hungernden Kontrolltieren, nachdem eine entsprechende Gewichtsabnahme eingetreten war) wurde ein Tryptophanbelastungstest durchgeführt. Dabei erhielt jedes Tier $40 \mathrm{mg}$ Tryptophan intraperitoneal in $4 \mathrm{~m} /$ 1 proz. Natriumbicarbonat-Lösung. Zur Uringewinnung wurden die Tiere für $24 \mathrm{Stdn}$. ohne Futter und Flüssigkeitszufuhr in röhrenförmigen Käfigen (12), in denen Kot und Harn getrennt voneinander aufgefangen werden können, gehalten. Der Harn wurde gemessen und die Xanthurensäurekonzentration bestimmt. Danach wurden die Tiere durch Genickschlag getötet, die Lebern sofort entnommen und in eiskalte 1 proz. KCl-Lösung gebracht. Gewebsproben von etwa $1 \mathrm{~g}$ wurden auf $50 \mathrm{mg}$ genau gewogen und in der Kühlkammer $\left(+2^{\circ}\right)$ mit der 9fachen Menge mit Triäthanolamin $(0,01 \mathrm{M}, \mathrm{pH} 7,4)$ gepufferter, 1 proz. $\mathrm{KCl}$-Lösung, die $0,5 \mathrm{mMol} / l \mathrm{EDTA}$ enthielt, versetzt, und im Homogenisator nach PotTER-ElveHJEM unter Eiskühlung homogenisiert. In den Leberhomogenaten wurden die Aktivitäten von Asparat- und Alaninaminotransferase ${ }^{2}$ ) bestimmt.

\section{Bestimmung von Xantburensäure nach (13)}

Der während 24 Stdn. ausgeschiedene Rattenharn wurde zentrifugiert und jeweils $1 \mathrm{~m} /$ des Zentrifugats mit $4 \mathrm{~m} /$ bidest. Wasser und $5 \mathrm{~m} / 0,4 \mathrm{M}$ Trispuffer ( $\mathrm{pH} 7,8$ ), der $0,4 \mathrm{Mol} / l$ Maleinat enthielt, versetzt. $5 \mathrm{~m} l$ dieser Lösung wurden mit $50 \mu l$ einer $1,7 \mathrm{proz}$. Eisenammoniumsulfatlösung (12-Hydrat) versetzt. Die Extinktion wurde nach $5 \mathrm{Min}$. bei $610 \mathrm{~nm}$ in $1 \mathrm{~cm}$ OS-Küvetten gegen die restliche Mischung (ohne Eisen) gemessen. Die der Extinktion entsprechende Xanthurensäuremenge wurde der Eichkurve entnommen.

Zur Anfertigung der Eichkurve wurden $10,0 \mathrm{mg}$ Xanthurensäure mit Âthanol und wenig 1N Ammoniak in Lösung gebracht und mit Äthanol auf $25,0 \mathrm{~m} l$ aufgefüllt. Durch entsprechende Verdünnung mit 5 proz. Athanol wurden die gewünschten Konzentrationen hergestellt und mit diesen die Farbreaktion wie oben durchgeführt.

2) Der Trivialname Aspartataminotransferase wird hier gebraucht für das Enzym L-Aspartat: 2-Oxo-glutarat Aminotransferase (EC 2.6.1.1); Alaninaminotransferase für L-Alanin: 2-Oxo-glutarat Aminotransferase (EC 2.6.1.2); I-Kynureninaminotransferase für L-Kynurenin: 2-Oxoglutarat Aminotransferase (EC 2.6.1.7); 3-Hydroxykynureninaminotransferase für 3 Hydroxykynurenin: 2-Oxoglutarat Aminotransferase (EC 2.6.1); Lactatdehydrogenase für L-Lactat: NAD Oxydoreductase (EC 1.1.1.27); Malatdehydrogenase für L-Malat: NAD Oxydoreductase (EC 1.1.1.37).
Bestimmung der Aktivitäten von Asparat- und Alaninaminotransferase in Anlehnung an (14)

Nach Vorinkubation von $100 \mu /$ Rattenleberhomogenat mit $0,9 \mathrm{~m} /$ $0,1 \mathrm{M}$ Triäthanolamin-Puffer $(\mathrm{pH} 7,4)$ und $0,5 \mathrm{~m} / 0,06 \mathrm{M}$ L-Asparaginsäure bzw. L-Alanin für $5 \mathrm{Min}$. bei $25^{\circ}$ wurde die Transaminasereaktion durch Zugabe von $0,5 \mathrm{~m} / 0,06 \mathrm{M} \alpha$-Ketoglutarsäurc gestartet und für weitere $10 \mathrm{Min}$. bei $25^{\circ}$ inkubiert. Die Reaktion wurde durch Zusatz von $2 \mathrm{~m} / 0,6 \mathrm{M}$ Perchlorsäure beendet; nach $10 \mathrm{Min}$. wurde zentrifugiert und $2 \mathrm{~m} /$ des Zentrifugats mit $2 \mathrm{~m} /$ $0,3 \mathrm{M} \mathrm{KHCO}_{3}$-Lösung neutralisiert. Das ausgefallene Kaliumperchlorat wurde in der Kühlkammer abfiltriert und in $2 \mathrm{~m} /$ des Filtrats Oxalacetat bzw. Pyruvat bestimmt. Bei den Leerwerten wurde statt der Substratlösungen ein entsprechendes Volumen Pufferlösung zugesetzt.

Die Bestimmung von Oxalessigsäure und Brenztraubensäure erfolgte im optischen Test nach (15) bzw. (16). Zu $2 \mathrm{~m} /$ Filtrat (entsprechend $2,5 \mathrm{mg}$ Leber) wurden in $1 \mathrm{~cm}$ OS-Küvetten $1 \mathrm{~m} / 0,4 \mathrm{M}$ Triäthanolamin-Puffer ( $\mathrm{pH} 7,6)$, der $0,04 \mathrm{Mol} / l$ EDTA enthielt, und $40 \mu / \mathrm{NADH}(14 \mathrm{mg} / \mathrm{m} /)$ gegeben. Nach Bestimmung der Anfangsextinktion wurde mit $20 \mu /$ Malatdehydrogenase $(0,5 \mathrm{mg} / \mathrm{ml})$ bzw. $20 \mu l$ Lactatdehydrogenase $(2 \mathrm{mg} / \mathrm{ml})$ gestartet und die Extinktionsdifferenz bei $366 \mathrm{~nm}$ ermittelt.

Die Leerwerte ergaben dabei Extinktionsdifferenzen unter 0,02, so daß sie unberücksichtigt bleiben konnten.

\section{Ergebnisse}

Körpergewvicht

Die Ratten, die L-Penicillamin erhielten, hatten während der Versuchsperiode im Mittel einen Gewichtsverlust von $10 \mathrm{~g}$, während die Tiere, die $\mathrm{D}$-Penicillamin erhielten, 4,5 $\mathrm{g}$ und die Kontrollen $12 \mathrm{~g}$ zunahmen (Tab. 1). Von 17 Ratten, die L-Penicillamin erhielten, starben 7 während der Versuchszeit innerhalb der ersten 10 Tage, wobei sie im Mittel $24 \mathrm{~g}$ an Gewicht verloren hatten. Die Ratten, die D-Penicillamin erhielten, und die Kontrollen erlebten alle das Ende der dreiwöchigen Versuchsperiode.

\section{Xantburensäure-Ausscheidung nach Tryptophanbelastung}

Die Kontrollen zeigen nach Tryptophanbelastung eine mittlere Xanthurensäure-Ausscheidung (Tab. 2) von $210 \mu \mathrm{g} / 24$ Stdn. Sie wird durch Verminderung des Körpergewichts infolge reduzierter Kost nicht beeinflußt (220 $\mu \mathrm{g} / 24$ Stdn.). Die Xanthurensäure-Ausscheidung der Tiere, die D-Penicillamin erhielten, betrug $810 \mu \mathrm{g} / 24$ Stdn. Der Unterschied ist signifikant $(p<0,01)$. Ungleich größere Mengen Xanthurensäure (im Mittel $5,9 \mathrm{mg} / 24 \mathrm{Stdn}$.) wurden von den Ratten nach L-Penicillamin-Gaben ausgeschieden. Diese Zahlenwerte entsprechen denen von Kuchrnskas und Mitarb. (3). Bei einer Ratte, die L-Penicillamin erhalten hatte, war die Xanthurensäureausscheidung nicht erhöht. Da intraperitoneale Injektionen in etwa 20\% der Fälle fehlinjiziert werden (17), kann eine ungenügende Tryptophanresorption z. B. aus Verdauungstrakt oder Blase dieses Ergebnis bedingen.

\section{Aktivität der Aspartataminotransfirase in der Leber}

Die Aktivität von Aspartataminotransferase (Tab.3) ist nur nach Gaben von L-Penicillamin signifikant vermindert, sie beträgt dann im Mittel 12,91 U/g Frischgewicht, während nach D-Penicillamin die gleichen Aktivitäten wie bei den Kontrolltieren gefunden werden (im Mittel 16,44 bzw. 16,85 U/g Frischgewicht). 
Tab. 1

Körpergewichte der Ratten

\begin{tabular}{|c|c|c|c|c|}
\hline & $\begin{array}{c}\stackrel{1}{\text { Kontrolle }} \\
\text { (Futter nach Belieben) }\end{array}$ & $\begin{array}{c}\text { II } \\
\text { Kontrolle } \\
\text { (Futter redzuiert) }\end{array}$ & $\begin{array}{c}\text { III } \\
\text { D-Penicillamin } \\
\text { (21. Tage, } 10 \mathrm{mg} \text { tgl.) }\end{array}$ & $\begin{array}{c}\text { IV } \\
\text { L-Penicillamin } \\
\text { (21 Tage, } 10 \mathrm{mg} \text { tgl.) }\end{array}$ \\
\hline $\begin{array}{l}\text { Anzahl der Versuchstiere } \\
\text { mittleres Anfangsgewicht (g) } \\
\text { m.ittleres Endgewicht }(\mathrm{g}) \\
\text { m.ittlere Differenz }(\mathrm{g})\end{array}$ & $\begin{array}{r}8 \\
249,0 \\
261,0 \\
+12,0 \pm 1,6\end{array}$ & $\begin{array}{r}7 \\
248,6 \\
229,3 \\
-19,3 \pm 1,3\end{array}$ & $\begin{array}{l}10 \\
256,2 \\
260,7 \\
+4,5 \pm 3,2\end{array}$ & $\begin{array}{l}10 \\
265,7 \\
255,9 \\
-9,8 \pm 2,6\end{array}$ \\
\hline
\end{tabular}

Tab. 2

Xanthurensäure-Ausscheidung der Fatten im 24-Stdn.-Harn nach Belastung mit 40 mg Tryptophan intraperitoneal

\begin{tabular}{|c|c|c|c|c|}
\hline & $\begin{array}{c}\text { I } \\
\text { Kontrolle } \\
\text { (Futtern nach Belieben) }\end{array}$ & $\begin{array}{c}\text { II } \\
\text { Kontrolle } \\
\text { (Futter reduziert) }\end{array}$ & $\begin{array}{c}\text { III } \\
\text { D-Penicillamin } \\
\text { (21 Tage, } 10 \mathrm{mg} \text { tgl.) }\end{array}$ & $\begin{array}{c}\text { IV } \\
\text { L-Penicillamin } \\
\text { (21 } \text { Tage, } 10 \mathrm{mg} \text { tgl.) }\end{array}$ \\
\hline $\begin{array}{l}\text { Anzahl der Versuchstiere } \\
\text { Mittelwerte (mg) }\end{array}$ & $0,21^{10} \pm 0,04$ & $0,22^{7} \pm 0,02$ & $\stackrel{10}{0,81} \stackrel{ \pm}{ \pm} 0,07$ & $\stackrel{10}{5,90 \stackrel{ \pm}{ \pm} 1,14}$ \\
\hline
\end{tabular}

Tab. 3

Transaminaseaktivität in den Lebern der Ratten

\begin{tabular}{|c|c|c|c|c|}
\hline (Fu & $\begin{array}{l}\text { I } \\
\text { Kontrolle } \\
\text { tter nach Belieben) }\end{array}$ & $\begin{array}{c}\text { II } \\
\text { Kontrolle } \\
\text { (Futter reduziert) }\end{array}$ & $\begin{array}{c}\text { III } \\
\text { (21 Tage, } 10 \mathrm{mg} \text { tgl.) }\end{array}$ & $\begin{array}{c}\text { IV } \\
\text { (21 Tage, } 10 \mathrm{mg} \text { tgl.) }\end{array}$ \\
\hline \multicolumn{5}{|c|}{ Aspartataminotransferase } \\
\hline $\begin{array}{l}\text { Anzahl der Versuchstiere } \\
\text { Mittelwerte ( } U / \mathrm{g} \text { Frischgewicht) }\end{array}$ & $16,85^{10} \pm 1,11$ & $21,92^{7} \pm 1,07$ & $16,44^{10} \pm 0,72$ & 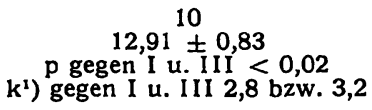 \\
\hline \multicolumn{5}{|c|}{ Alaninaminotransferase } \\
\hline $\begin{array}{l}\text { Anzahl der Versuchstiere } \\
\text { Mittelwerte (U/g Frischgewicht) }\end{array}$ & $16,01^{10} \pm 0,88$ & $18,16^{7} \pm 0,76$ & $\begin{array}{c}13,63 \stackrel{10}{ \pm 0,82} \\
\text { pegen I }<0,1 \\
\text { k gegen } 12,0\end{array}$ & $\begin{array}{c}10 \\
\begin{array}{c}8,65 \\
\text { p gegen I } \\
\text { u. III } 1,09\end{array}<0,01 \\
\text { gegen I u. III } 5,3 \text { bzw. } 3,7\end{array}$ \\
\hline
\end{tabular}

1) $\mathrm{k}$ wui de nach der Formel $\mathrm{k}=\frac{M_{1}-M_{2}}{\sqrt{\varepsilon_{1}^{2}+\varepsilon_{2}^{2}}}$ berechnet. $\quad M=$ Mittelwert $\quad \varepsilon \doteq$ mittlere Abweichung des Mittelwertes

\section{Aktivität der Alaninaminotransferase in der Leber}

Die Aktivität von Alaninaminotransferase (Tab. 3) ist auch nach Gaben von D-Penicillamin (im Mittel 13,63 U/g Frischgewicht) gegenüber den Kontrollen (im Mittel 16,01 U/g Frischgewicht) etwas vermindert ( $p$ $<0,1)$. Nach L-Penicillamin ist die Alaninaminotransferase-Aktivität mit im Mittel 8,65 U/g Frischgewicht erheblich vermindert $(p<0,01)$. Sie ist auch gegenüber den Tieren, die D-Penicillamin erhielten, signifikant erniedrigt $(p<0,01)$. Die Zahlenwerte für die Kontrolltiere entsprechen den Befunden anderer Autoren $(18-20)$.

\section{Diskussion}

Nach diesen Ergebnissen bestehen also ganz wesentliche Unterschiede in der Antivitamin-B $\mathrm{B}_{6}$-Wirkung der beiden Antipoden von Penicillamin. Das ist besonders deswegen bemerkenswert, da beide Penicillamine mit dem vom Vitamin $\mathrm{B}_{6}$ hergeleiteten Coenzym, dem Pyridoxalphosphat, gleich schnell $\mathrm{zu}$ Thiazolidinen reagieren (21, 22). Auch bei den Wirkungen der Penicillamine, die nicht als Antivitamin- $B_{6}$-Wirkung aufgefaßt werden können, bestehen Unterschiede zwischen den Antipoden. Diese sind jedoch nicht Gegenstand unserer Untersuchungen.

Heddle und Mitarb. (23) wiesen darauf hin, daß die toxischen Wirkungen von L-Penicillamin durch Vitamin- $B_{6}-Z_{\text {Zlagen nicht }}$ vollständig verhindert oder aufgehoben werden können. WACKER und Mitarb. (24) fanden, daß L-Penicillamin im Gegensatz zur D-Form bei der zellfreien Proteinsynthese in Proteine eingebaut werden kann. Unterschiede einer unspezifischen toxischen Wirkung können so gedeutet werden.
Daß tatsächlich eine spezifische Antivitamin-Wirkung besteht und eine solche nicht als Folge der bei L-Penicillamingaben beobachteten Gewichtsabnahme der Versuchstiere vorgetäuscht wird, zeigen auch die Versuche mit den Tieren, die infolge reduzierten Futterangebots in 5 Tagen im Mittel $20 \mathrm{~g}$ abgenommen hatten, aber nach Tryptophanbelastung normale Xanthurensäure-Ausscheidung zeigten. Die Transaminaseaktivitäten in der Leber waren bei diesen Tieren sogar gesteigert. Eine Erhöhung von Transaminaseaktivitäten bei Hungertieren wurde beschrieben (19) und als Folge einer katabolen Stoffwechsellage angesehen (25).

Die erhöhte Xanthurensäure-Ausscheidung nach Tryptophanbelastung zeigt eine unterschiedliche Empfindlichkeit der am Tryptophanstoffwechsel beteiligten von Pyridoxalphosphat abhängigen Enzyme gegenüber Penicillamin an. Diese entspricht dem Verhalten dieser Enzyme bei Pyridoxin-Mangel (26). Die Aktivitätsminderung von Enzymen, die Reaktionen katalysieren, die auf dem Wege vom Tryptophan zur Anthranilsäure, 3-Hydroxyanthranilsäure und Nicotinsäureamid liegen, sowie der L-Kynureninaminotransferase, deren Aktivität für die Bildung von Kynurensäure entscheidend ist, muß wesentlich stärker sein als die von 3-Hydroxykynureninaminotransferase, deren Wirkung zur Bildung von Xanthurensäure führt. Der Tryptophanbelastungstest ist also ein $\mathrm{Maß}$ für die relative Hemmung verschiedener Pyridoxalphosphat-abhängiger Enzyme des Tryptophanstoffwechsels (27-29).

Einen großen Unterschied der Empfindlichkeit verschiedener Pyridoxalphosphat-Enzyme gegen die Penicill- 
amin-Antipoden zeigen auch die Transaminasebestimmungen in der Leber. Während die Konzentration der Aspartataminotransferase in der Leber durch D-Penicillamin gar nicht, durch L-Penicillamin nur geringfügig vermindert wird, ist die Konzentration der Alaninaminotransferase auch nach D-Penicillamin vermindert $(20 \%)$ und wird durch L-Penicillamin erheblich gesenkt (50\%).
Über vergleichende Untersuchungen der Wirkung der Penicillaminantipoden und ihrer Kondensationsprodukte mit Pyridoxalphosphat auf isolierte Enzyme werden wir in einer weiteren Mitteilung berichten (30).

Fräulein Brigitte Clemens ( $\dagger$ ) danken wir für ihre stets zuverlässige technische Assistenz.

\title{
Literatur
}

1. Wilson, J. E. und V. Du Vigneaud, Science (New York) 107, 635 (1948). - 2. Wilson, J. E. und V. du Vigneaud, J. biol. Chemistry 184, 63 (1950). - 3. Kuchinskas, E. J. und V. DU Vigneaud, Arch. Biochem. Biophysics 66, 1 (1957). - 4. KuchinsKas, E. J., A. Horvath und V. DU Vigneaud, Arch. Bioctem. Biophysics 68, 69 (1957) - 5. DU Vigneaud, V., E. J. KuCHINSKas und A. Horvath, Arch. Biochem. Biophysics 69, 130 (1957). 6. Asatoor, A. M., Nature (London) 203, 1382 (1964). - 7. JAFFE, I. A., K. Altman und P. Merryman, J. Clin. Invest. 43, 1869 (1964) - 8. GrbBs, K. und J. M. WALSHE, Lancet (London) I, 175 (1966). - 9. BeNESCH, R. und R. E. BeNESCH, Determination of -SH Groups in Proteins; in: Methods of Biochemical Analysis, Bd. X, S. 43. Hrsg. D. Glick, Interscience Publishers, Inc. New YorkLondon (1962); Boyer, P. D., J. Amer. chem. Soc. 76, 4331 (1954). - 10. Schlossmann, K., J. BrügGemann und F. Linen, Biochem. Z. 336, 258 (1962). - 11. Toknnies, G. und R. P. Homiller, J. Amer. chem. Soc. 64, 3054 (1942) - 12. Herken, H., G. SenFT und H. Winurzkr, Naunyn-Schmiedebergs Arch. exp. Pathol. Pharmakol. 229, 123 (1956). - 13. WACHSTEIN, M. und A. GuDartis, Amer. J. Clin. Path. 22, 652 (1952). - 14. Weber, F. und O. Wiss, Hoppe-Seylers Z. physiol. Chem. 331, 124 (1963) - 15. Hoнorst, H.-J. und M. REIM, Oxalacetat, in: Methoden der enzymatischen Analyse, Hrsg. H.-U. Bergmeyer, S. 535, Verlag Chemie GmbH, Weinheim/Bergst. (1962). - 16. Bücher, TH., R. Czok, W. Lamprecht und E. Latzko, Pyruvat, in: Methoden der enzymatischen Analyse, Hrsg. H.-U. Bergmeyer, S. 253, Verlag Chemie GmbH, Weinheim/Bergstr. (1962). - 17. Lewrs, R. E., A. L. Kunz und R. E. Bell, Laborat. Animal Care 16, 505 (1966). - 18. Persson, B. H., Acta Soc. med. Upsal. 65, 96 (1960). - 19. ThIele, V. F. und M. Brin, J. Nutrit. 90, 347 (1966). - 20. Chatterjee, A. K., S. C. Jamdar und B. B. Ghosh, Experientia, Basel 22, 794 (1966). - 21. Siegmund, P., diese Z. 1, 97 (1963). 22. Siegmund, P., F. Körber und G. Hasenaank, diese Z. 4, 307 (1966). - 23. Hedde, J. G., E. W. McHenry und G. H. Beaton, Canad. J. Biochem. 41, 1215 (1963). - 24. WACKER, A., P. Chandra und E. Heyl, Arzneimittel-Forsch., Aulendorf 16, 825 (1966). - 25. Rorzsch, W., Acta biol. med. german. 16, 329 (1966). - 26. KörnER, W. F. und H. Nowak, Int. Z. Vitaminforsch. 36, 264 (1966). - 27. Hughes, P. A. M. und D. N. Raine, Clin. chimica Acta (Amsterdam) 14, 399 (1966). - 28. Coon, W. W., Amer. J. Clin. Path. 46, 345 (1966). - 29. KöRNER, W. F. und H. NowaK, Int. Z. Vitaminforsch. 37, 89 (1967). - 30. Hasenbank, G., F. Körber und P. Siegmund, Hoppe-Seylers Z. physiol. Chem. im Druck und in Vorbereitung.

Privat-Dozent Dr. P. Siegmund 1 Berlin 33, Arnimallee 22

\section{Über die Mg-Aufnahme in Erythrocyten und Ascites-Tumorzellen}

\author{
Von H. EbeL und TH. GüNTHER \\ Aus dem Physiologisch-Chemischen Institut der Freien Universität Berlin (Direktor: Prof. Dr. Dr. E. Scbütte)
}

(Eingegangen am 20. Juli 1967)

An glykolysierenden Menschenerythrocyten, atmenden Vogelerythrocyten und glykolysierenden und atmenden Ascites-Tumorzellen wurde die $\mathrm{Mg}$-Aufnahme bei einer auf 6 bis $7 \mathrm{mMol} / \mathrm{l}$ erhöhten extrazellulären $\mathrm{Mg}$-Konzentration untersucht.

Alle untersuchten Zellen nehmen mit 1,5 mMol/l Zellen gleichviel $\mathrm{Mg}$ auf.

Die Mg-Aufnahme ist nicht durch einfache Diffusion bedingt und setzt sich aus Adsorption an die Zelloberfläche und stoffwechselabhängiger $\mathrm{Mg}$-Aufnahme in die Zelle zusammen.

Die Mg-Aufnahme wird durch Glucose und Phosphàt gefördert und durch Phlorrhizin und Arsenat gehemmt.

Die Mg-Aufnahme ist ATP-abhängig und wird durch Strophanthin-g gehemmt.

Steigende $\mathrm{Na}^{+}-\mathrm{Konzentrationen} \mathrm{fördern} \mathrm{die} \mathrm{Mg}$-Aufnahme bis zu einem Maximum bei physiologischen $\mathrm{Na}^{+}-\mathrm{Konzentrationen.} \mathrm{Bei}$ physiologischer $\mathrm{Na}^{+}$-Konzentration liegt das Maximum der die $\mathrm{Mg}$-Aufnahme fördernden $\mathrm{K}^{+}$-Konzentration bei 7,5 mMol/l; höhere $\mathrm{K}^{+}-$Konzentrationen wirken hemmend.

Es wird diskutiert, daß die membrangebundene $\mathrm{Mg}^{2+}-\mathrm{Na}^{+}-\mathrm{K}^{+}-\mathrm{AT}^{-}$Pase an der $\mathrm{Mg}-\mathrm{Aufnahme}$ beteiligt ist.

The uptake of $\mathrm{Mg}$ by glycolysing human erythrocytes, respiring bird erythrocytes, and glycolysing and respiring Ascites tumor cells was studied at increased levels $(6-7 \mathrm{mM})$ of extracellular $\mathrm{Mg}$.

In all the cells, the uptake of $\mathrm{Mg}$ was the same, i. e., $1.5 \mathrm{mmoles} / \mathrm{l}$ cells.

The uptake of $\mathrm{Mg}$ is not the result of simple diffusion; it is due to the combined effects of adsorption onto the cell surface and the metabolic-dependent uptake of $\mathrm{Mg}$ into the cell.

The uptake of $\mathrm{Mg}$ is promoted by glucose and phosphate and inhibited by phlorrhizin and arsenate.

The uptake of $\mathrm{Mg}$ is ATP-dependent and is inhibited by strophanthin-g.

Increasing concentrations of $\mathrm{Na}^{+}$promote the uptake of $\mathrm{Mg}$ to a maximum corresponding to the physiological concentration of $\mathrm{Na}^{+}$. At physiological concentrations of $\mathrm{Na}^{+}$, the optimum concentration of $\mathrm{K}^{+}$for the promotion of $\mathrm{Mg}$-uptake was $7.5 \mathrm{mM}$; higher concentrations of $\mathrm{K}^{+}$were inhibitory.

It is suggested that the membrane-bound $\mathrm{Mg}^{2+}-\mathrm{Na}^{+}-\mathrm{K}^{+}-\mathrm{ATPase}$ is involved in the uptake of $\mathrm{Mg}$. 